\title{
Construindo mapas cognitivos de jogos digitais: um estudo de caso com o jogo Pac-Man
}

\author{
Luciana R. Guedes $^{1}$, Marcelo da S. Hounsell ${ }^{1}$, Aleksander S. Paterno ${ }^{2}$ \\ ${ }^{1}$ Departamento de Ciência da Computação \\ Universidade do Estado de Santa Catarina \\ ${ }^{2}$ Departamento de Engenharia Elétrica \\ Universidade do Estado de Santa Catarina
}

R. Paulo Malschitzki, 200 - Zona Industrial Norte - 89219-710 - Joinville - SC - Brasil

\{luciana.guedes, marcelo.hounsell, aleksander.paterno\}eudesc.br

\begin{abstract}
A cognitive map is a tool for specify what cognitive aspects are trigged when a human task is performed. It is generally used to evaluate cognitive functions related to pedagogical activities or to perform psycopedagogical diagnostic. It can be applied to any activity or even to a game. Then, this game could be used for detecting impairments in cognitive funcions on players. This work presents a proposal to develop cognitive maps related to a digital game, describing its conception stage and, later, its validation by specialists. This case study is applied to the classical digital game Pac-Man.
\end{abstract}

Resumo. Um mapa cognitivo é uma ferramenta usada para caracterizar uma atividade humana em termos de quais aspectos cognitivos são desencadeados com tal atividade. São geralmente utilizados para avaliar funções cognitivas em atividades pedagógicas ou em diagnósticos psicopedagógicos, mas podem ser aplicados a outras tarefas, até mesmo a um jogo. Neste caso, o jogo pode ser utilizado para indicar deficiências em funções cognitivas dos jogadores. Neste artigo é apresentada a proposta de desenvolvimento de mapas cognitivos para caracterização de um jogo digital detalhando sua etapa de concepção e, posteriormente, a validação por especialistas. Como estudo de caso, é proposta a aplicação deste método no jogo digital clássico Pac-Man.

\section{Introdução}

O uso de jogos digitais em situações que vão além do entretenimento tem ocorrido em diversas áreas da atividade humana, como é o caso da educação. O conceito de jogo digital é de "um embate mental, jogado com um computador de acordo com certas regras, para diversão, recreação ou para ganhar uma aposta" [Zyda 2005].

Para ilustrar o interesse dos pesquisadores na aprendizagem baseada em jogos digitais, constatou-se que, somente nos anais do Simpósio Brasileiro de Informática na Educação do ano de 2020, foram encontrados 36 artigos completos (cerca de 20\%) que mencionam o uso de jogos aplicados a contextos educacionais. Destes 36 artigos, destacase que 24 deles referem-se a jogos sérios. Os jogos sérios são aqueles criados originalmente para atender propósitos específicos (contextos sérios), sem desprezar seu objetivo lúdico [Alvarez and Djaouti 2011]. No entanto, é comum usar jogos criados com 
o propósito de entretenimento ("não-sérios") como suporte para outras áreas, ou seja, com propósito "sério". A expressão aprendizagem baseada em jogos digitais indica a utilização de jogos em contextos educacionais [Prensky 2001]. A proposta do presente trabalho utiliza um jogo digital clássico ("não-sério") para avaliar aspectos cognitivos de seus jogadores.

Do ponto de vista de aspectos cognitivos, a teoria da Experiência da Aprendizagem Mediada (EAM) oferece ferramentas que podem apoiar estratégias para detectar deficiências cognitivas, bem como para corrigir estas deficiências [Feuerstein et al. 2002]. A teoria da EAM modela, de forma sistemática, a interação do sujeito com os estímulos externos e permite o desenvolvimento de meta-cognição nos aprendizes. O trabalho de Castro e Brito (2002) apresenta uma proposta do uso da EAM em alunos com deficiência mental num ambiente informatizado [Castro and da Silva Brito 2002]. Raabe e Giraffa (2006) utilizam os preceitos da EAM numa proposta de Inteligência Artificial Aplicada a Educação, em sistemas tutores inteligentes [Raabe and Giraffa 2006].

Uma das ferramentas da EAM é o mapa cognitivo, que possibilita compreender os processos mentais envolvidos numa atividade [Feuerstein et al. 1988]. Ele é comumente utilizado para apoiar atividades pedagógicas ou até avaliações psicopedagógicas, sendo o instrumento que fundamenta o processo de análise. A partir do mapa cognitivo, que indica os processos cognitivos de uma atividade, é possível avaliar o desempenho de uma pessoa ao executar tal atividade. A criação de um mapa cognitivo não é uma tarefa trivial pois requer uma análise que tem um grau de subjetividade, além do conhecimento específico da área.

O objetivo deste trabalho é o de apresentar uma proposta de mapa cognitivo do jogo Pac-Man usando os parâmetros da EAM para caracterizá-lo e, posteriormente, validar esta proposta com a contribuição de especialistas em desenvolvimento destes mapas cognitivos. Esta análise irá contribuir para enquadrar as práticas de interpretação de jogos digitais através das lentes da teoria da Modificabilidade Cognitiva Estrutural, da teoria da EAM e de seus aspectos correlacionados sob o ponto de vista neurocientífico. O resultado deste trabalho servirá como fundamento para atividades posteriores de avaliação de funções cognitivas deficientes a partir deste jogo digital. A obtenção de um mapa cognitivo que seja validado por especialistas pode servir como base para outros estudos a partir de uma referência bem consistente.

\section{Mapas Cognitivos de Reuven Feuerstein}

Reuven Feuerstein foi um psicólogo e pesquisador do século XX (1921-2014). Entre as suas contribuições está a teoria da Modificabilidade Cognitiva Estrutural (MCE) [Feuerstein et al. 2015], que preconiza que o ser humano pode aprimorar sua capacidade de aprendizagem em qualquer idade, até mesmo aqueles que possuem ou vivenciaram condições desfavoráveis na infância. A proposta relaciona-se com a teoria de Experiência da Aprendizagem Mediada (EAM), que usa a mediação como elemento-chave para o processo de aprendizagem [Feuerstein et al. 1991]. A mediação é uma forma de interação humana que visa fazer o aprendiz pensar como os estímulos/informações são processados par aa solução dos problemas. Feuerstein teve a oportunidade de comprovar sua teoria com crianças que enfrentaram situações extremas como órfãos de guerra ou com algumas cuja capacidade cognitiva considerada como limitada, como crianças do Síndrome 
de Down [Feuerstein et al. 2015].

No contexto da teoria da EAM, um mapa cognitivo é "uma ferramenta conceitual que descreve as dimensões de uma tarefa" que pode ser utilizado para apoiar a avaliação das dificuldades de aprendizagem de um indivíduo [Feuerstein et al. 2015]. A rigor, qualquer atividade humana pode ser caracterizada como uma tarefa a partir do momento que exige um esforço mental (ainda que seja pequeno) para ser executada. No contexto da EAM, um mapa cognitivo descreve os atos mentais necessários para realização de uma tarefa específica, com base em sete parâmetros pelos quais estes atos mentais podem ser analisados, categorizados e ordenados. Estes parâmetros são divididos em três conjuntos: parâmetros contextuais, parâmetros operacionais e parâmetros modais.

Os parâmetros contextuais caracterizam a atividade em termos de quais conteúdos ela abrange e quais modalidades de linguagem são utilizadas. Os parâmetros operacionais estão relacionados às operações mentais e às funções cognitivas que precisam ser mobilizadas para que a tarefa seja realizada. Os parâmetros modais determinam os níveis de complexidade, abstração e eficiência exigidos pela tarefa que está sendo caracterizada no mapa cognitivo.

Assim, os parâmetros do ato mental fundamentam a construção dos mapas cognitivos. Estes, por sua vez, são ferramentas que dão suporte para a avaliação de desempenho cognitivo a partir de tarefas a serem executadas pelos sujeitos que serão avaliados. Estes parâmetros são descritos em detalhes a seguir [Feuerstein et al. 2002]:

- Conteúdo: Diz respeito ao assunto ao qual o ato mental se refere e ao universo de conteúdos em que ele opera;

- Modalidade: Refere-se a modalidade de linguagem utilizada na tarefa. Basicamente, pode ser: verbal, pictórica (desenhos,imagens), numérica, figurativa (simbólica). Pode ser também uma combinação de várias modalidades;

- Operações Mentais: Representam as operações que são necessárias para executar os atos mentais exigidos pela tarefa; [Gomes 2002] descreve 16 diferentes operações mentais: 1-identificação, 2-análise, 3-comparação, 4-síntese, 5-classificação, 6-seriação, 7-diferenciação, 8-codificação e decodificação, 9projeção de relações virtuais, 10-representação mental, 11-pensamento divergente, 12-pensamento lógico, 13-pensamento hipotético-inferencial, 14pensamento transitivo, 15-pensamento analógico e 16-pensamento silogístico. $\mathrm{Na}$ prática, as operações mentais atuam em conjunto com as funções cognitivas mas, para efeitos de construção do mapa cognitivo, elas são consideradas separadamente;

- Fase (funções cognitivas): Um ato mental pode ser dividido em três diferentes fases: entrada, elaboração e saída. Aqui, busca-se selecionar qual a fase de pensamento predominante na qual a execução da tarefa está focada. Para cada uma das fases, existe um conjunto de funções cognitivas associadas, conforme apresentado em detalhes na Figura 1;

- Nível de Complexidade: Pode ser entendido em termos de quantidade e qualidade de unidades de informação contidas no ato mental;

- Nível de Abstração: Define a distância entre o ato mental da tarefa em relação ao objeto ou evento no qual ele opera;

- Nível de Eficiência: Diz respeito ao nível de eficiência exigido para a execução da 
tarefa. Pode ser expresso em termos de tempo, produtividade, número de acertos, acurácia, entre outras medidas, seja quantitativa ou qualitativamente.

$\mathrm{Na}$ proposta original do autor, a construção de mapas cognitivos apoiam as estratégias de condutas de mediação para aprimoramento de aspectos cognitivos identificados como deficientes. O presente trabalho faz parte de uma proposta mais abrangente do uso de jogos digitais para detecção destas deficiências.

\subsection{Funções Cognitivas Deficientes}

As funções cognitivas são definidas como pré-requisitos para cada processo do pensamento que fornece qualidade para o ato mental [Feuerstein 1997]. Elas podem ser expressas na forma positiva, em termos de "habilidades" (mentais) que são necessárias para a operacionalização de uma ação. No entanto, o objetivo da construção de mapas cognitivos costuma ser a descoberta das "dificuldades" do sujeito de modo a buscar seu aprimoramento. Assim, é mais comum expressar as funções cognitivas "deficientes" durante a construção deste mapas. A EAM classifica as funções cognitivas em termos três fases: entrada, elaboração e saída. As funções de entrada referem-se a problemas ocorridos na captação da informação vinda do mundo exterior ao sujeito. As funções de elaboração dizem respeito ao modo como ele processa as informações. Por fim, as funções de saída estão relacionadas às limitações do sujeito para expressar os resultados de seus pensamentos. A Figura 1 apresenta a lista das funções cognitivas em sua forma deficiente.

Quando um indivíduo está realizando uma atividade para solucionar um problema, há necessidade de mobilizar as funções cognitivas. Quando isto não ocorre adequadamente, a solução do problema fica comprometida, o que pode caracterizar funções cognitivas deficientes. A teoria da EAM propõe intervenções de mediação para atuar sobre as funções cognitivas, tornando-as eficientes. Em sala de aula, o papel de mediação cabe primordialmente ao professor. No entanto, a mediação pode ocorrer a partir de qualquer indivíduo que auxilie usando os critérios de mediação descritos por Feuerstein.

A EAM pode ser usada na apresentação de qualquer conteúdo ou atividade. Neste trabalho, pretende-se especificar e aplicar estes conceitos em jogos digitais através da construção de mapas cognitivos que caracterizam estes jogos e, com isto, permitem a descoberta de funções cognitivas deficientes do jogadores durante as partidas.

\subsection{Trabalhos Relacionados}

Os mapas cognitivos desenvolvidos a partir da proposta de Feuerstein são encontrados na literatura, principalmente em trabalhos voltados à educação ou à pscicologia. Além das propostas do próprio criador destes mapas, como em [Feuerstein et al. 1991] e [Feuerstein et al. 1991], também há citações destes instrumentos em trabalhos como [Avendaño and Parada-Trujillo 2012], onde o mapa é apresentado em forma de tabela como proposta de ferramenta para identificar funções cognitivas deficientes em estudantes num contexto de sala de aula. Já em [Gomes 2002], o autor apresenta vários exemplos de atividades com uma análise das funções cognitivas por elas mobilizadas. Embora faça considerações clínicas sobre cada atividade apresentada, o autor não chega a descrevêlas com todos os parâmetros de um mapa cognitivo. Estes trabalhos não efetuaram a validação dos mapas cognitivos propostos mediante a avaliação de especialistas. 


\section{Funções de Entrada}

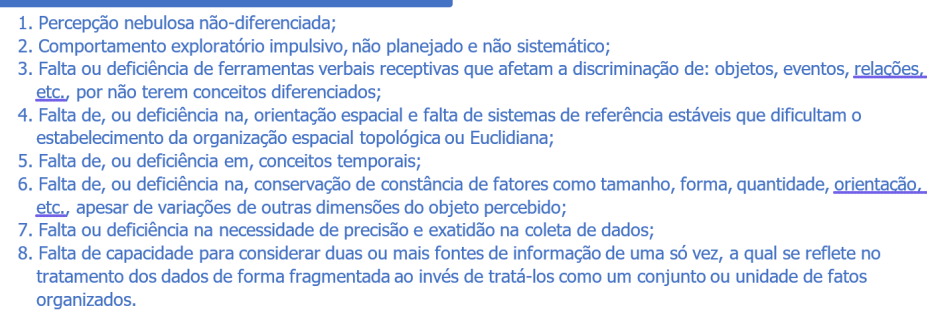

\section{Funções de Elaboração}

9. Percepção inadequada da existência de um problema e em sua definição

10. Incapacidade para selecionar/diferenciar dados relevantes de não-relevantes na definição de um problema;

11. Falta de comportamento comparativo espontâneo ou limitação de sua aplicação por um sistema de necessidade restrito;

12. Limitação do campo mental

3. Percepção episódica da realidade, em que as experiências são vivenciadas como únicas e isoladas

14. Falta ou deficiência de necessidade de busca de evidências lógicas que orientem a resolução de problemas;

15. Falta ou deficiência de interiorização;

16. Falta de, ou deficiência no, pensamento hipotético-inferencial

17. Falta ou deficiência de estratégias para comprovar hipótese

18. Falta ou deficiência no comportamento de planejamento;

. Não elaboraçâo de certas categorias cognitivas quando conceitos verbais não fazem parte do repertório do indivíduo em nível receptivo ou expressivo.

\section{Funções de Saída}

20. Uso de modalidades de comunicação egocêntrica;

21. Dificuldade na projeção de relações virtuais;

22. Bloqueio (mental);

23. Respostas por tentativa e erro;

24. Falta ou deficiência de ferramentas verbais para comunicar adequadamente as respostas elaboradas

25. Falta ou deficiência de necessidade de precisão e correção na comunicação de respostas;

26. Dificuldade na transposição de uma imagem visual, de uma representação de um contexto a outro.

27. Conduta de resposta impulsiva.

\section{Figura 1. Funções Cognitivas Deficientes segundo a teoria da EAM.}

Fonte: Adaptado de [Feuerstein et al. 1991]

Considerando que este trabalho está relacionado com jogos digitais, foram encontradas algumas propostas de modelagem de jogos com este tipo mapa cognitivo. Este é o caso do trabalho de Soeira (2011), que apresentou a proposta de um mapa cognitivo do jogo The Sims, no formato de sete itens (cada item correspondendo a um parâmetro do mapa) [Soeira 2011]. Embora este trabalho tenha relacionado o jogo com um mapa cognitivo, a autora utiliza este recurso apenas como forma de comparar o jogo analisado com jogos de empresa e para confirmar quais habilidades cognitivas são demandadas. $\mathrm{O}$ trabalho não aprofunda estes conceitos e os parâmetros do mapa cognitivo não são testados de modo formal nem validado por especialistas.

Ainda na temática de jogos, mas numa abordagem de jogos de tabuleiro voltados ao ensino da Matemática, Silva (2013) apresenta um exemplo de mapa cognitivo dentro de um modelo de tabela, usando o jogo do Tangram ${ }^{1}$ [Silva 2013]. Reforça-se aqui que este trabalho não apresenta um jogo digital e sim, um jogo de tabuleiro. Além disso, a autora tem como foco a utilização do mapa para atividades de sala de aula e também não usa método formal (como o apoio de especialistas, por exemplo) para construção ou na validação deste mapa. Este modelo foi usado como base para construção do mapa cognitivo de jogos no presente trabalho, com seu formato tabular que abrange todos os

\footnotetext{
${ }^{1} \mathrm{O}$ Tangram é "um passatempo de origem chinesa, composto de sete peças (cinco triângulos, um quadrado e um paralelogramo) que se montam como um puzzle para formar diversas figuras". [Priberam 2020]
} 
parâmetros originais de Feuerstein.

\section{Proposta para Mapa Cognitivo em Jogo Digital}

Embora a maioria das pesquisas com jogos digitais em contextos educacionais utilizem jogos sérios, este trabalho traz a proposta de uso de um jogo digital clássico, construído para fins de entretenimento, para apoiar a detecção de funções cognitivas deficientes.

O método escolhido para o presente trabalho segue os seguintes passos: 1) apresentação das características do jogo escolhido; 2) apresentação de como foi feito o mapa cognitivo do jogo pelos autores; 3) apresentação do mapa cognitivo elaborado a partir dos especialistas e 4) análise dos resultados.

\subsection{Escolha do Jogo Pac-Man}

O jogo selecionado para o estudo de caso foi o Pac-Man, um jogo clássico de labirinto do tipo Arcade, desenvolvido originalmente no Japão pela empresa Namco in 1980. A versão escolhida neste trabalho é uma versão para computador que roda em um ambiente web, ou seja, utiliza o navegador (browser) de internet. Trata-se de um jogo do tipo single player (não é jogado contra outros oponentes), não imersivo (o jogador não se sente dentro do ambiente do jogo) e bidimensional (usa apenas as direções vertical e horizontal). Os jogadores podem apenas usar o teclado para mover o personagem do jogo em um dos sentidos: para cima, para baixo, para esquerda ou para direita.

A imagem apresentada na Figura 2 mostra a aparência do jogo Pac-Man usado neste trabalho.

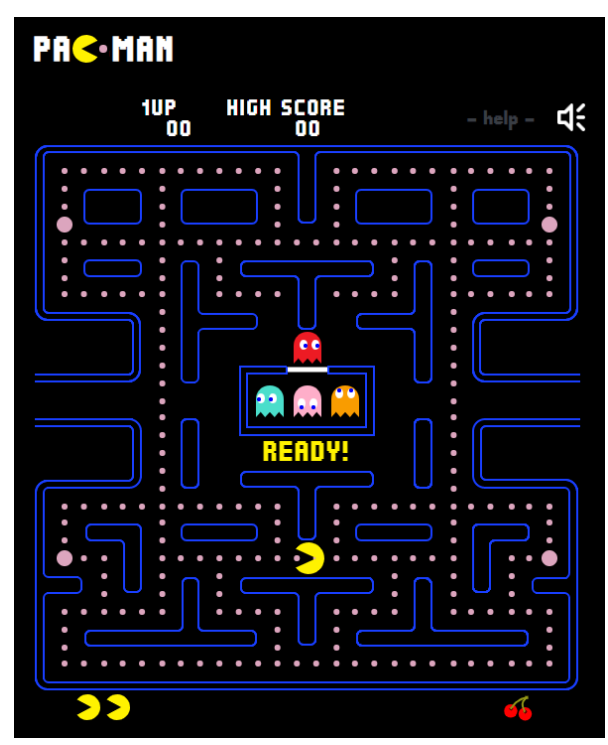

Figura 2. Tela do jogo Pac-Man.

Fonte: Reprodução da tela inicial do Jogo Pac-Man.

O objetivo do jogo é conduzir o personagem principal, conhecido como Pac-Man, por um labirinto pré-definido para coletar todas as bolinhas e pastilhas enquanto tenta esquivar-se de quatro fantasmas que tentam persegui-lo. O jogador inicia a partida com 3 vidas. Se o Pac-Man é apanhado por qualquer um dos fantasmas, ele perde uma vida 
e retorna ao ponto inicial. Uma partida termina quando todos as bolinhas e pastilhas são apanhadas (vitória) ou quando o Pac-Man perde todas as vidas (derrota). Há 240 bolinhas e 4 pastilhas espalhadas ao longo do labirinto. Cada bolinha dá ao jogador 10 pontos, enquanto cada pastilha garante 50 pontos. As pastilhas são bolinhas maiores distribuídas em quatro extremidades do labirinto. Ao coletar estas pastilhas, além de conquistar 50 pontos, o Pac-Man passa a ter o poder temporário de perseguir e matar os fantasmas, que tornam-se azuis e passam a fugir do Pac-Man, ao invés de caçá-lo. Nesta situação, quando os fantasmas são apanhados, eles dão ao jogador, respectivamente, 200, 400, 800 e 1600 pontos.

A escolha deste jogo foi motivada pelas características mais restritas de sua jogabilidade de modo que torna-se mais assertiva a avaliação de ações específicas dos jogadores tais como teclas pressionadas, direção escolhida, velocidade de respostas, entre outras.

\subsection{Mapa Cognitivo do Jogo Pac-Man}

Após a definição do jogo que seria utilizado, a etapa seguinte foi a criação do Mapa Cognitivo usando os sete parâmetros do ato mental.

A criação de mapas cognitivos conforme idealizado por Feuerstein (1980) é uma atividade geralmente realizada por especialistas com formação específica, que é o Programa de Enriquecimento Instrumental (PEI), de responsabilidade do ICELP (Centro Internacional para o Desenvolvimento do Potencial de Aprendizagem) [Feurstein 1980]. Não é limitado a psicólogos, embora muitos destes especialistas tenham formação também em psicologia.

A criação do mapa cognitivo do jogo foi possível a partir do estudo da Teoria da EAM e da experiência de usar o jogo por diversas vezes (apesar do jogo ter uma mecânica simples, estima-se que são necessárias, pelo menos, três partidas para extrairse os parâmetros cognitivos associados a ele). A Tabela 1 apresenta, em negrito, alguns parâmetros estabelecidos no mapa cognitivo desenvolvido nesta etapa. A seguir, alguns destes parâmetros relativos à fase (funções cognitivas deficientes) são esclarecidos:

\section{Funções Cognitivas de Entrada:}

- Falta ou deficiência de orientação espacial (o personagem principal movimenta-se em quatro direções pelo uso das setas do teclado);

- Falta ou deficiência de conceitos temporais (é necessário um grau de previsibilidade temporal em relação ao tempo que os fantasmas podem se aproximar do personagem principal e destruí-lo);

- Falta de capacidade para considerar duas ou mais fontes de informação de uma só vez (necessária para gerenciar a movimentação simultânea dos quatro fantasmas e do deslocamento do personagem principal).

\section{Funções Cognitivas de Elaboração:}

- Percepção episódica da realidade, em que as experiências são vivenciadas como únicas e isoladas (é necessário que o jogador compreenda o contexto geral do jogo, o que já foi completado e o que ainda falta realizar para ganhar o jogo);

- Falta ou deficiência no comportamento de planejamento (o planejamento das ações durante a partida é um requisito relevante).

Funções Cognitivas de Saída: 
- Conduta de resposta impulsiva (a impulsividade pode levar o jogador a perder eficiência com ações desnecessárias, como tentar pressionar a mesma tecla repetidamente, o que não tem efeito algum neste jogo).

A partir das justificativas de escolha dos parâmetros que apresentadas anteriormente, é possível perceber a associação existente entres as mecânicas do jogo e as funções cognitivas demandadas para jogá-lo. No entanto, a atividade de construção destes mapas costuma ser realizada por especialistas com formação específica. Assim, a etapa seguinte foi a de buscar a validação do mapa a partir de pessoas com esta formação e com experiência nesta tarefa.

\subsection{Mapa Cognitivo Criado por Especialistas}

A criação de mapas cognitivos é geralmente realizada por especialistas com formação no PEI. Esta formação pode ser dada a qualquer pessoa, mas é comumente voltada a profissionais como psicólogos, pedagogos e neuropsicólogos. A experiência na criação de mapas cognitivos é um fator que contribui para a confiabilidade no resultado do desenvolvimento deste instrumento.

Sendo assim, como parte da metodologia deste trabalho, foram consultados especialistas com formação no PEI e com experiência no desenvolvimento de mapas cognitivos para que pudessem apresentar sua proposta pessoal de mapa cognitivo para o jogo digital Pac-Man. Os especialistas consultados não tiveram acesso à proposta preliminar aqui apresentada, mas apenas construíram sua versão pessoal, com base em sua percepção e na experiência de jogar o jogo o número de vezes que considerassem suficiente. Esta etapa foi conduzida a partir de projeto protocolado junto ao Comitê de Ética em Pesquisa com Seres Humanos sob número CAAE 44051121.1.0000.0118.

Nesta etapa, foram convidados cinco especialistas com formação no PEI e com experiência de mais de cinco anos no desenvolvimento de mapas cognitivos. O perfil dos especialistas consultados (em termos de formação e experiência) aparece na Figura 3. Aqui cabe esclarecer que a criação de mapas cognitivos está sujeita a um grau de subjetividade e seus resultados depende da avaliação de cada especialista. Por este motivo, a experiência do especialista torna-se relevante. Outro aspecto importante é a compreensão da tarefa que, neste caso, corresponde a entender como o jogo funciona.

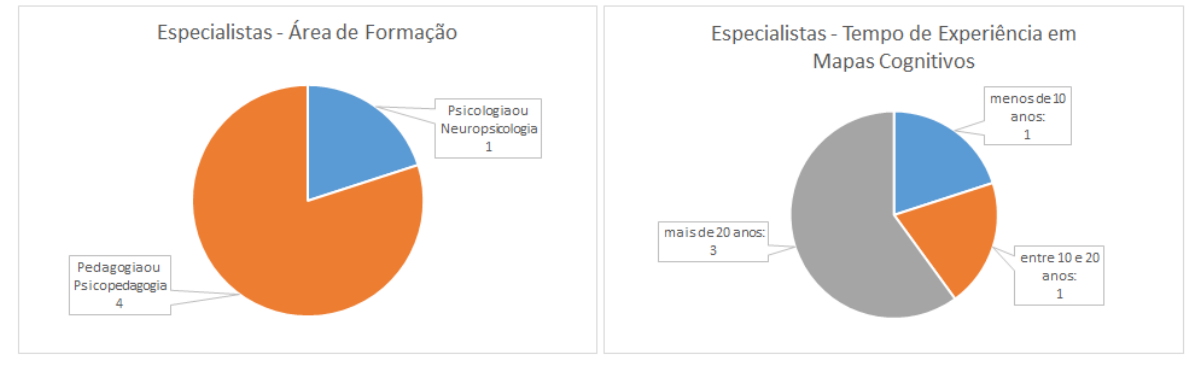

Figura 3. Perfil dos especialistas consultados.

Fonte: Elaborado pelos autores.

Após a coleta de dados através de formulário, as informações foram compiladas de modo a construir um único mapa convergente com as propostas individuais. Para isto, 
em atributos de múltipla escolha foram selecionados os itens mais votados por todos, considerando-se apenas aqueles que tiveram pelo menos três votos. A Tabela 1 apresenta estes atributos com um valor proporcional (porcentagem) de indicações por parte dos especialistas. $\mathrm{O}$ atributos que aparecem com destaque em negrito nesta mesma tabela indicam valores que coincidiram com a proposta feita pelos autores.

Tabela 1. Mapa Cognitivo do Pac-Man segundo especialistas - compilação.

\begin{tabular}{|c|c|c|}
\hline \multicolumn{3}{|c|}{ MAPA COGNITIVO - JOGO PAC-MAN (por especialistas) } \\
\hline Nome do Jogo: & \multicolumn{2}{|c|}{ Jogo Pac-Man para Web. } \\
\hline Conteúdo & \multicolumn{2}{|c|}{ Jogo digital Pac-Man. } \\
\hline Modalidade & \multicolumn{2}{|c|}{$\begin{array}{l}\text { - pictórica }(80 \%) \\
\text { - simbólica }(60 \%)\end{array}$} \\
\hline $\begin{array}{l}\text { Operações } \\
\text { Mentais }\end{array}$ & \multicolumn{2}{|c|}{$\begin{array}{l}\text { - projeção de relações virtuais }(100 \%) \\
\text { - codificação e decodificação }(80 \%) \\
\text { - análise }(60 \%) \\
\text { - comparação }(60 \%) \\
\text { - representação mental }(60 \%) \\
\text { - pensamento hipotético-inferencial }(60 \%)\end{array}$} \\
\hline \multirow{3}{*}{$\begin{array}{l}\text { Fases / } \\
\text { Funções } \\
\text { Cognitivas }\end{array}$} & Entrada & $\begin{array}{l}\text {-Falta ou deficiência de orientação espacial (100\%) } \\
\text {-Comportamento exploratório impulsivo, não plane- } \\
\text { jado e não sistemático ( } 80 \%) \\
\text {-Falta ou deficiência de conceitos temporais }(80 \%) \\
\text {-Percepção nebulosa não-diferenciada }(60 \%) \\
\text {-Falta de capacidade para considerar duas ou mais } \\
\text { fontes de informação de uma só vez }(60 \%)\end{array}$ \\
\hline & Elaboração & $\begin{array}{l}\text {-Percepção inadequada da existência de um problema } \\
\text { e em sua definição }(60 \%) \\
\text {-Incapacidade para selecionar/diferenciar dados rele- } \\
\text { vantes de não-relevantes }(60 \%) \\
\text {-Falta de comportamento comparativo espontâneo } \\
(60 \%) \\
\text {-Percepção episódica da realidade, em que as ex- } \\
\text { periências são vivenciadas como únicas e isoladas } \\
(60 \%) \\
\text {-Falta ou deficiência no comportamento de plane- } \\
\text { jamento }(60 \%)\end{array}$ \\
\hline & Saída & $\begin{array}{l}\text {-Respostas por tentativa e erro }(100 \%) \\
\text {-Conduta de resposta impulsiva }(80 \%)\end{array}$ \\
\hline Nível de Complexid. & \multicolumn{2}{|c|}{ Médio-baixo [valor ponderado=1,71] } \\
\hline Nível de Abstração & \multicolumn{2}{|c|}{ Baixo [valor ponderado=1,95] } \\
\hline Nível de Eficiência & \multicolumn{2}{|c|}{ Médio-alto [valor ponderado=3,52] } \\
\hline
\end{tabular}

Fonte: Elaborado pelos autores com dados dos especialistas, 2021.

No caso dos três último atributos, que são de alternativa única, foi usada uma ponderação com base no tempo de experiência dos especialistas, atribuindo-se peso maior aos que tinham maior tempo, mediante normalização. Para isto, as respostas (baixo, médio-baixo, médio, médio-alto e alto) foram convertidas em valores inteiros de 1 a 5 , sendo atribuído o valor 1 para a opção "baixo"e o valor 5 para a opção "alto". Para a normalização foi escolhido o método "min-max", que mostrou-se mais adequado devido 


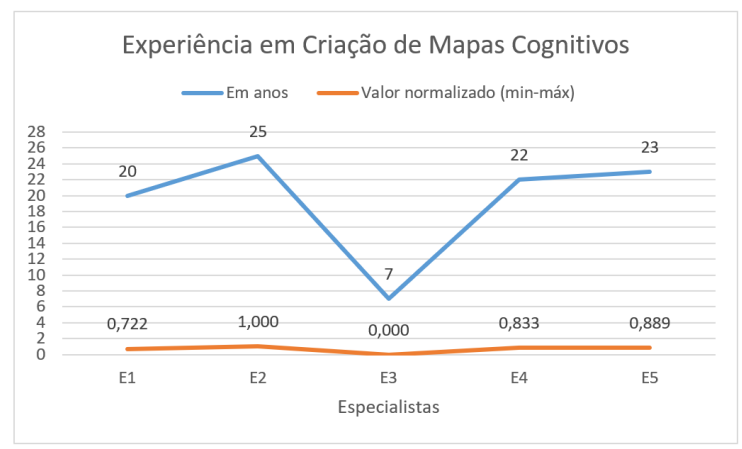

Figura 4. Experiência dos especialistas em criação de mapas cognitivos.

Fonte: Elaborado pelos autores.

a existência de um outlier. Os valores nominais e normalizados aparecem no gráfico da Figura 4.

O tempo de experiência dos especialistas com seu valor normalizado foi usado como ponderação para os três últimos parâmetros. Este valor numérico resultante da média ponderada é mostrado na Tabela 1 entre colchetes. Para se chegar no resultado, a média ponderada obtida foi arredondada para um valor inteiro e convertida numa das cinco opções (baixo, médio-baixo, médio, médio-alto ou alto), usando-se a mesma ideia anterior de correspondência de 1 a 5 , respectivamente.

\section{Considerações Finais}

Este trabalho apresentou uma proposta de desenvolvimento de mapa cognitivo de um jogo digital com base na teoria da EAM. O jogo escolhido foi o Pac-Man clássico e, após o mapa gerado, foi feita a validação por especialistas no desenvolvimento destes mapas.

Após a compilação dos resultados foi possível perceber que a proposta preliminar de mapa cognitivo elaborada pelos autores teve muitos pontos em comum com a proposta compilada a partir das opiniões dos especialistas. Esta afirmação pode ser verificada pelos atributos apresentados em negrito na Tabela 1. Os parâmetros "modalidade", "operações mentais" e "fases/funções cognitivas" tiveram, pelo menos, um item em comum com a proposta dos autores e vários deles tiveram dois ou mais itens em comum, o que evidencia esta convergência. Quanto aos três últimos parâmetros, tanto o "nível de complexidade" quanto o "nível de eficiência" coincidiram com a proposta dos autores. Já no parâmetro "nível de abstração", o resultado foi diferente da proposta dos autores (que tinham escolhido a opção 'baixo'). No entanto, salienta-se que este também foi o item mais divergente mesmo entre os especialistas (40\% escolheram a opção 'baixo', outros $40 \%$ escolheram 'médio-baixo' e a opção 'médio' foi escolhida por $20 \%$ ).

O desenvolvimento do mapa cognitivo de um jogo abre possibilidades para sua utilização em atividades pedagógicas ou em situações de avaliação psicopedagógicas. Em trabalhos futuros, pretende-se utilizar os parâmetros apresentados no mapa cognitivo para avaliar funções cognitivas deficientes dos jogadores. O próximo desafio será relacionar, de modo automatizado, os dados oriundos das partidas jogadas com os parâmetros estabelecidos no mapa cognitivo de modo a investigar funções cognitivas deficientes. 


\section{Referências}

Alvarez, J. and Djaouti, D. (2011). An introduction to serious game definitions and concepts. In Serious Games \& Simulation for Risks Management, volume 11, p. 11-15.

Avendaño, W. R. and Parada-Trujillo, A. E. (2012). Cognitive map in the process of learning assessment. Investigación y Desarrollo, 20(2):334-365.

Castro, R. and da Silva Brito, G. (2002). Avaliando os alunos com deficiência mental educável através do método de reuven feuerstein em ambiente informatizado. In Brazilian Symposium on Computers in Education (Simpósio Brasileiro de Informática na Educação-SBIE), volume 1, p. 192-199.

Feuerstein, R. (1997). The coherence of the theory of modifiability, p. 29-36. ICELP Publications.

Feuerstein, R., Feuerstein, R., and Falik, L. H. (2015). Beyond smarter: Mediated learning and the brain's capacity for change. Teachers College Press.

Feuerstein, R., Feuerstein, R. S., Falik, L. H., and Rand, Y. (2002). The dynamic assessment of cognitive modifiability: The Learning Propensity Assessment Device: Theory, instruments and techniques, Rev. and exp. ed. of The dynamic assessment of retarded performers. ICELP Publications.

Feuerstein, R., Klein, P. S., and Tannenbaum, A. J. (1991). Mediated learning experience (MLE): Theoretical, psychosocial and learning implications. Freund Publishing House Ltd.

Feuerstein, R., Rand, Y., and Rynders, J. E. (1988). Don't accept me as I am: helping "retarded"people to excel. Springer Science+Bussiness Media.

Feurstein, R. (1980). Instrumental enrichment: An intervention program for cognitive modifiability. University Park Press.

Gomes, C. M. A. (2002). Feuerstein e a construção mediada do conhecimento. Artmed.

Prensky, M. (2001). Digital Game-Based Learning. McGraw-Hill.

Priberam (2020). Dicionário Priberam da Língua Portuguesa [em linha].

Raabe, A. L. A. and Giraffa, L. M. M. (2006). Uma arquitetura de tutor para promover experiências de aprendizagem mediadas. In Brazilian Symposium on Computers in Education (Simpósio Brasileiro de Informática na Educação-SBIE), volume 1, p. 278287.

Silva, S. (2013). A intencionalidade educativa nos jogos matemáticos. PhD thesis, Escola Superior de Educadores na Infância.

Soeira, E. d. R. (2011). Competências e habilidades cognitivas em jogos eletrônicos de simulação. In X Congresso Nacional de Educação - EDUCERE, p. 10444-10457.

Zyda, M. (2005). From visual simulation to virtual reality to games. Computer, 38(9):2532. 